\title{
Review of: "Psychometric evaluation of patient assessment of chronic illness care among Korean cancer survivors"
}

\author{
Ahmad Farouk Musa ${ }^{1}$
}

1 Monash University

Potential competing interests: The author(s) declared that no potential competing interests exist.

1.Thank you very much for inviting me to prepare a review of the manuscript Psychometric evaluation of patient assessment of chronic illness care among Korean cancer survivors. I found the manuscript to be very well-written and the results were well-analysed.

2.The main aim of this study was to examine the psychometric properties, validity, and reliability of a Korean version of the Patient Assessment of Chronic Illness Care (PACIC) which has been translated into Korean and conducted among cancer survivors (K-PACIC-CS). Authors have shown that the psychometric properties of the K-PACIC-CS to be a valid and reliable instrument for measuring Chronic Care Management model from the survivor's perspective. In this study, the authors used PACIC translated by Kim YE and performed the back to back translation and adapted some questionnaires to the oncology care setting.

3.The results of the study are presented in a transparent way. The Cronbach's alpha value of the K-PACICCS was very high and convergent validity was established, and the CFA was acceptable. In addition, the authors were aware of the limitations of research, such as conducting a test-retest which encourages research to continue. The authors also pointed to the practical application of research results. While the low rate of missing data was explained, the authors should also discussed about the merit and demerit of conducting the interview in this manner.

4.My final comment is in providing the responsiveness of the questionnaire or the Minimal clinically important differences (MCID) which would reflect the grade of the validity of the questionnaire to detect the changes in a clinical intervention in cancer patients. Without these evidence, it would be difficult to claim that these questionnaires are objectively useful in a clinical setting for physicians to identify changes in patients' health. 